\title{
Surface-associated lipoprotein PpmA of Streptococcus pneumoniae is involved in colonization in a strain-specific manner
}

\author{
Correspondence \\ P. W. M. Hermans \\ P.Hermans@cukz.umcn.nl
}

Received 15 December 2008

Revised 25 March 2009

Accepted 26 March 2009
L. E. Cron, ${ }^{1}$ H. J. Bootsma, ${ }^{1}$ N. Noske, ${ }^{2}$ P. Burghout, ${ }^{1}$ S. Hammerschmidt ${ }^{2}$ and P. W. M. Hermans ${ }^{1}$

\author{
${ }^{1}$ Laboratory of Pediatric Infectious Diseases, Radboud University Nijmegen Medical Centre, \\ Nijmegen, The Netherlands \\ ${ }^{2}$ Department Genetics of Microorganisms, Ernst Moritz Arndt University of Greifswald, Greifswald, \\ Germany
}

\begin{abstract}
Streptococcus pneumoniae produces two surface-associated lipoproteins that share homology with two distinct families of peptidyl-prolyl isomerases (PPlases), the streptococcal lipoprotein rotamase $A(S \mid r A)$ and the putative proteinase maturation protein $A(P p m A)$. Previously, we have demonstrated that SIrA has PPlase activity, and that the enzyme plays a role in pneumococcal virulence. Here, we investigated the contribution of PpmA to pneumococcal pathogenesis. Pneumococcal mutants of D39 and TIGR4 lacking the gene encoding PpmA were less capable of persisting in the nasopharynx of mice, demonstrating the contribution of PpmA to pneumococcal colonization. This observation was partially confirmed in vitro, as the pneumococcal mutants NCTC10319 $\Delta p p m A$ and TIGR4 $\Delta c p s \Delta p p m A$, but not D39 $\Delta c p s \Delta p p m A$, were impaired in adherence to Detroit 562 pharyngeal cells. This suggests that the contribution of PpmA to pneumococcal colonization is not solely the result of its role in adherence to epithelial cells. Deficiency in PpmA did not result in reduced binding to various extracellular matrix and serum proteins. Similar to SlrA, we observed that PpmA was involved in immune evasion. Uptake of PpmA-deficient D39 $\Delta$ cps and NCTC10319 by human polymorphonuclear leukocytes was significantly enhanced compared to the isogenic wild-types. In addition, ingestion of D39 $\Delta p p m A$, but not that of either NCTC10319 $\Delta p p m A$ or TIGR4 $\Delta p p m A$, by murine macrophage cell line J774 was also enhanced, whereas intracellular killing remained unaffected. We conclude that PpmA contributes to the early stages of infection, i.e. colonization. The contribution of PpmA to virulence can be explained by its strain-specific role in adherence to epithelial cells and contribution to the evasion of phagocytosis.
\end{abstract}

\section{INTRODUCTION}

Streptococcus pneumoniae resides in the nasopharyngeal cavity of the human host. Pneumococcal carriage is regarded as the key linking transmission of this Grampositive bacterium to other susceptible individuals, but is also considered to be a prerequisite for disease. Although colonization is usually asymptomatic, it can progress to disease when colonizing S. pneumoniae infiltrate the middle ear, lungs, brain or blood. Consequently, this pathogen is a major cause of morbidity and mortality worldwide, especially in young children, the elderly and immunocompromised individuals (Bogaert et al., 2004).

Abbreviations: $\mathrm{Cl}$, competitive index; FCS, fetal calf serum; hplgR, human polymeric immunoglobulin receptor; $\mathrm{PMN}$, polymorphonuclear leukocyte; PPlase, peptidyl-prolyl isomerase.
Multiple bacterial factors are required for colonization and infection of the host to occur. For instance, the expression of capsule reduces entrapment in the luminal mucus, allowing the pneumococci access to epithelial surfaces (Nelson et al., 2007). Inside the host, this polysaccharide capsule serves as a biochemical barrier, protecting the pneumococcus from phagocytosis and killing by inhibiting complement deposition and consequently complement activation (Hammerschmidt et al., 2005). Several bacterial adhesins are involved in adherence to the epithelial surface, including phosphorylcholine (ChoP), which interacts with the receptor for platelet-activating factor (rPAF) (Cundell et al., 1995; Swords et al., 2000). In addition, ChoP serves as a non-covalent anchor for a group of surface proteins known as the choline-binding proteins. Of these, PspC (also referred to as CbpA or SpsA), binds to human secretory component (SC) of the polymeric Ig receptor 
during the first stage of translocation across the epithelium (Elm et al., 2004; Hammerschmidt, 2006; Zhang et al., 2000).

In addition to the surface components directly involved in adhesion and immune invasion, factors influencing proper protein folding and surface composition are also essential for bacterial virulence. Peptidyl-prolyl isomerases (PPIases) are ubiquitous foldases, which accelerate the cis-trans conformational changes at the Xaa-Pro bonds during protein folding (Hermans et al., 2006; Rahfeld et al., 1994). They are classified into three separate families, i.e. the parvulins, the FK506-binding proteins (FKBP) and the cyclophilins. The parvulins are the smallest known proteins with PPIase activity, and have a lower relative activity against biological substrates than the cyclophilins and the FK506-binding proteins. PPIases have been identified in both eukaryotes and prokaryotes. For example, SurA is a parvulin located in the periplasm of Escherichia coli which has a role in the assembly of outer-membrane proteins of Gram-negative bacteria (Lazar \& Kolter, 1996; Rouviere \& Gross, 1996). Another parvulin is PrsA, which is a membrane-bound lipoprotein known to be involved in the protein export and maturation machinery of Grampositive bacteria (Vitikainen et al., 2004). The product of a prsA-like gene from Lactococcus lactis, PmpA, is thought to possess foldase activity facilitating protein secretion (Drouault et al., 2002). Several studies have also indicated a role for PPIases in virulence. For instance, the trigger factor of Streptococcus pyogenes is considered to be responsible for the secretion of many virulence factors (Lyon et al., 1998). The macrophage infectivity potentiator (Mip) protein of Legionella pneumophila belongs to the FKBPs and is required for entry into human alveolar macrophages and intracellular replication of this Gramnegative micro-organism (Cianciotto et al., 1989; Cianciotto \& Fields, 1992).

The pneumococcal genome contains four putative PPIases, two of which are produced as surface-associated lipopro- teins: the streptococcal lipoprotein rotamase A (SlrA) and the putative proteinase maturation protein $\mathrm{A}(\mathrm{PpmA})$. In a previous study, we demonstrated that SlrA is a functional cyclophilin-type PPIase involved in pneumococcal colonization, as $s l r A$ mutants are rapidly eliminated from the upper airways in a mouse pneumonia model. In addition, we provided evidence that a deficiency in SlrA substantially reduces adherence to and internalization by epithelial and endothelial cells (Hermans et al., 2006).

PpmA is the second surface-associated PPIase, and is abundantly produced and conserved among pneumococci. It has homology to the parvulin family of proteins, but no detectable PPIase activity has been found to date (Hermans et al., 2006; Overweg et al., 2000a). It was previously shown that $p p m A$-deficient mutants display reduced virulence in a mouse pneumonia model (Overweg et al., 2000a). In the current study, we investigated the role of PpmA in pneumococcal virulence in more detail. We show here that PpmA contributes to pneumococcal colonization of the murine nasopharynx. Furthermore, we demonstrate that PpmA is involved in in vitro adherence to epithelial cells and that expression of PpmA interferes with uptake by professional phagocytes.

\section{METHODS}

Pneumococcal strains and growth conditions. Pneumococcal strains used in this study are shown in Table 1. S. pneumoniae was routinely grown in Todd-Hewitt broth supplemented with $5 \mathrm{~g}$ yeast extract $1^{-1}$ (THY) or on Columbia blood agar (BA) plates (Oxoid) at $37{ }^{\circ} \mathrm{C}$ and $5 \% \mathrm{CO}_{2}$. The number of c.f.u. per millilitre in a particular sample was quantified by plating serial 10 -fold dilutions on BA plates. When indicated, antibiotics were used at the following concentrations: kanamycin, $250 \mu \mathrm{g} \mathrm{ml}{ }^{-1}$, trimethoprim, $25 \mu \mathrm{g} \mathrm{ml}{ }^{-1}$ and streptomycin $100 \mu \mathrm{g} \mathrm{ml}^{-1}$.

Construction of pneumococcal mutants. The D39 $\Delta p p m A$ and NCTC10319 $\triangle p p m A$ mutants were constructed by in-frame insertion deletion mutagenesis using the trimethoprim resistance cassette, $d f r 13$, as described previously (Hermans et al., 2006). To obtain the

Table 1. Bacterial strains and mutants used in this study

\begin{tabular}{|lcll|}
\hline Strain & Serotype & Antibiotic resistance & \multicolumn{1}{c|}{ Reference } \\
\hline D39 wild-type & 2 & & NCTC 7466 \\
D39:: rpsL & 2 & Streptomycin & This study \\
D39 $\Delta p p m A$ & 2 & Trimethoprim & Hermans et al. $(2006)$ \\
D39 $\Delta$ cps & - & Kanamycin & This study \\
D39 $\Delta p p m A \Delta c p s$ & - & Trimethoprim, kanamycin & This study \\
NCTC10319 wild-type & $35 \mathrm{~A}$ & & Hermans et al. $(2006)$ \\
NCTC10319:: $r p s L$ & $35 \mathrm{~A}$ & Streptomycin & This study \\
NCTC10319 $\Delta p p m A$ & $35 \mathrm{~A}$ & Trimethoprim & Hermans et al. $(2006)$ \\
TIGR4 wild-type & 4 & & Tettelin et al. $(2001)$ \\
TIGR4 $:: r p s L$ & 4 & Streptomycin & This study \\
TIGR4 $\Delta p p m A$ & 4 & Trimethoprim & This study \\
TIGR4 $4 c p s$ & - & Kanamycin & This study \\
TIGR4 $\Delta p p m A \Delta c p s$ & - & Trimethoprim, kanamycin & This study \\
\hline
\end{tabular}


TIGR4 $\triangle p p m A$ strain, a $\triangle p p m A-P C R$ product was amplified from D39 $\triangle$ ppmA with primer pair HBppmAF1 (5'-CTCTTGATGGCTGAACATGC-3') and HBppmAR1 (5'-GCAGCCTACAGCTAGCTTCC- $\left.3^{\prime}\right)$. This PCR product was introduced into TIGR4 by transformation, and trimethoprim-resistant colonies were checked for the $p p m A$ mutation by PCR.

The isogenic capsule locus ( $c p s$ ) deletion mutants were constructed by allelic replacement of the entire capsule gene cluster with the kanamycin resistance cassette of plasmid pR410 (Burghout et al., 2007) as follows. Primers HBdexBF3 (5'-TATCAGGAAGGTCAGCCTAA-3') and HBdexBR2_F4 (5'-TAAGCTTGATATCGAATTCCTGCACTAAGGACACGCTTCT- $3^{\prime}$ ), and HBaliAF_R (5'-GGATGAATTGTTTTAGTACCTGACGCGCTTACGATATTCA-3') and HBaliAR ( $5^{\prime}$-TTTCGCAAATTCAACAGAGG-3'), were used to generate PCR products of the left (dexB) and right (aliA) flanking regions of the cps locus (approx. $900 \mathrm{bp}$ each). These PCR products were fused to the kanamycin resistance gene amplified with primers HBkanF4 (5'-GGAATTCGATATCAAGCTTA-3') and HBkanR (5'AGGTACTAAAACAATTCATCC-3') by means of overlap extension PCR. The resulting PCR product was transformed into S. pneumoniae, and kanamycin-resistant transformants were checked for the presence of the $c p s$ deletion by PCR. The $\Delta p p m A \Delta c p s$ double mutants were constructed by transformation of the $\Delta c p s$-PCR product (amplified with primer pair HBdexBF3 and HBaliAR) to each $\triangle p p m A$ mutant and selecting for trimethoprim and kanamycin resistance. Resulting transformants were checked for the $p p m A$ and cps deletions by PCR.

To obtain the streptomycin-resistant D39::rpsL, NCTC10319:: rpsL and TIGR $4:: r p s L$ strains, the $r p s L$ gene encoding a streptomycinresistant mutant of the ribosomal protein S12 was amplified from D39:: rpsL (Hermans et al., 2006) with primer pair HBrpsLF (5'-GTACAGGGACGTGCTGACAA-3') and HBrpsLR (5'CCCTTTCCTTATGCTTTTGG-3'). This PCR product was introduced into D39, NCTC10319 and TIGR4 by transformation, and streptomycin-resistant transformants were checked for the rpsL mutation by sequence analysis.

Mouse colonization model: single infection. Prior to mouse infection experiments, bacteria were passaged in mice to maintain virulence as described previously (Kerr et al., 2004). Cultures of mouse-passaged S. pneumoniae strains were grown to an $\mathrm{OD}_{620}$ of 0.25 and stored in aliquots at $-80{ }^{\circ} \mathrm{C}$ in $15 \%$ glycerol. Prior to infection, aliquots of bacteria were spun down and were resuspended in sterile PBS. In the colonization model, female 8-week-old CD-1 mice were lightly anaesthetized with $2.5 \%(\mathrm{v} / \mathrm{v})$ isoflurane over oxygen $\left(1.51 \mathrm{~min}^{-1}\right)$. Ten microlitres containing $5 \times 10^{6}$ c.f.u. of bacteria was distributed over both nostrils while the mice were held vertically by the scruff of the neck. Because such a low volume is given, only the nasopharynx of the mice becomes infected. Groups of five or six mice were sacrificed at $0.5,24,48,96$ and $192 \mathrm{~h}$ postinfection. Bacteria were recovered from the nasopharynx by flushing each nare with $1 \mathrm{ml}$ sterile PBS (Hendriksen et al., 2008) and plated for c.f.u. count. In addition, the lungs from each mouse were removed from the body, homogenized in $2 \mathrm{ml}$ sterile PBS using a hand-held homogenizer (Polytron PT 1200, Kinematica AG), and plated. The 30 min time point is considered to be the start of the infection, and is therefore referred to as $t=0$.

Mouse colonization model: co-infection. For the co-infection, a $10 \mu \mathrm{l}$ inoculum containing a $1: 1$ ratio of streptomycin-resistant wildtype and trimethoprim-resistant $p p m A$ mutants $\left(5 \times 10^{6}\right.$ c.f.u. total $)$ was used to infect female 8 -week-old CD-1 mice intranasally as described above. This model reduces variation between individual mice, inoculation preparation and distribution, and sample collection. Groups of five or six mice were sacrificed at 0.5, 24, 48, 96 and $192 \mathrm{~h}$ post-infection. Samples from nasopharynx and lungs were collected as described above. Again, the $30 \mathrm{~min}$ time point is considered to be the start of the infection, and is therefore referred to as $t=0$. Bacteria recovered from the nasopharynx were plated onto BA plates and Columbia agar plates supplemented with $5 \%(\mathrm{v} / \mathrm{v})$ defribrinated sheep blood (Biotrading) containing either streptomycin or trimethoprim to determine c.f.u. counts. Competitive index (CI) scores were calculated for the co-infection experiments as previously described (Hava \& Camilli, 2002). In short, a CI score was obtained by dividing the output ratio of the c.f.u. counts of mutant to wild-type by the input ratio of mutant to wild-type bacteria. For experiments in which no mutant bacteria were recovered from a particular mouse, the lower limit of detection (i.e. 20 c.f.u. $\mathrm{ml}^{-1}$ ) was substituted as the numerator. A CI score of 1 indicates no change in the ratio of wild-type and mutant before and after infection. Values lower than 1 indicate that the mutant is out-competed by the wildtype strain. All experiments were performed with approval of the Animal Experimentation Committee (DEC) of the Radboud University Nijmegen Medical Centre.

Cell lines and cell culture. The human pharyngeal epithelial cell line Detroit 562 (ATCC number CCL-138) was routinely grown in RPMI 1640 medium without phenol red (Invitrogen) supplemented with $1 \mathrm{mM}$ sodium pyruvate and $10 \%(\mathrm{v} / \mathrm{v})$ fetal calf serum (FCS). The murine macrophage-like cell line J774 (ATCC TIB 67) was cultured in Dulbecco's modified Eagle's medium (DMEM, PAA Laboratories) supplemented with $10 \%$ FCS, $2 \mathrm{mM}$ glutamine, penicillin $\mathrm{G}\left(100 \mathrm{IU} \mathrm{ml}^{-1}\right)$ and streptomycin $\left(100 \mu \mathrm{g} \mathrm{ml}^{-1}\right)$. All cells were cultured at $37^{\circ} \mathrm{C}$ in a $5 \% \mathrm{CO}_{2}$ environment.

Adherence assay. For adherence assays, bacteria were grown to mid-exponential phase in THY and stored in $1 \mathrm{ml}$ aliquots at $-80{ }^{\circ} \mathrm{C}$ in THY containing $15 \%$ glycerol. Before each assay, bacteria were resuspended in RPMI 1640 medium without phenol red supplemented with $1 \%$ FCS. Adherence of pneumococci to epithelial cells was performed as described previously (Bootsma et al., 2007; Hermans et al., 2006). Briefly, Detroit 562 cells were seeded into 24-well plates and incubated for $48 \mathrm{~h}$. Confluent monolayers were washed twice with PBS and infected with $1 \times 10^{7}$ c.f.u. $\mathrm{ml}^{-1}$ (m.o.i. of 10 bacteria per cell) and pneumococci were allowed to adhere to the cells for $2 \mathrm{~h}$ at $37{ }^{\circ} \mathrm{C}$ in a $5 \% \mathrm{CO}_{2}$ environment. Non-adherent bacteria were removed by three $1 \mathrm{ml}$ washes with PBS, after which $200 \mu \mathrm{l}$ of $25 \%$ trypsin and $1 \mathrm{mM}$ EDTA was added to detach the cells, followed by $800 \mu \mathrm{l}$ of ice-cold $0.025 \%$ Triton X-100 in PBS to lyse the cells. Samples were plated for c.f.u. count, and corrected to account for small differences in the initial inoculum count. All experiments were performed in triplicate and repeated at least three times. The adherence of the mutants is given as the percentage relative to the wild-type. Wild-type and mutant strains grew comparably in RPMI medium (without phenol red supplemented with $1 \%$ FCS) alone.

Binding of pneumococci to immobilized host proteins and extracellular matrix. Human factors such as laminin, fibronectin, fibrinogen, lactoferrin, type IV collagen, plasminogen, serum IgA, human secretory component and reconstituted basement membrane (Matrigel; BD Biosciences) were used to coat 96-well plates and kept overnight at $4{ }^{\circ} \mathrm{C}$. The proteins were purchased from commercial suppliers (Calbiochem, Sigma and Roche). The surface of the wells was then blocked with $1 \%$ BSA for at least $3 \mathrm{~h}$ at room temperature. Labelling of the bacteria with FITC was performed as described by Bergmann et al. (2003). Extensively washed FITC-labelled wild-type or ppmA-deficient pneumococci $\left(2.5 \times 10^{7}\right)$ were added to the wells and incubated for $1 \mathrm{~h}$ at $37^{\circ} \mathrm{C}$ to allow binding. Fluorescence was measured at $485 / 538 \mathrm{~nm}$ (excitation/emission) using a Fluoroskan Ascent (ThermoLabsystems). Binding is expressed as the percentage of the total applied FITC-labelled pneumococci bound to the immobilized proteins. Values are from a representative experiment 
and are the mean of triplicates. Each experiment was performed at least three times with similar results.

Phagocytosis of bacteria by granulocytes. Human polymorphonuclear leukocytes (PMNs) were isolated from peripheral blood of four healthy volunteers. Equal volumes of blood and PBS were mixed, layered on a Ficoll-Histopaque gradient, and centrifuged for $20 \mathrm{~min}$ at $800 \mathrm{~g}$. The isolated phase containing PMN cells was depleted of erythrocytes by two consecutive hypotonic shocks (Jansen et al., 1998). Finally, the PMNs were resuspended in RPMI 1640 (PAA Laboratories) and immediately used for the phagocytosis assay. PMNs were incubated at a density of $6 \times 10^{5} \mathrm{ml}^{-1}$ for $30 \mathrm{~min}$ in $50 \mu \mathrm{RPMI}$ $1640 / 1 \%$ FCS with FITC-labelled S. pneumoniae at an m.o.i. of 50 at $37^{\circ} \mathrm{C}$. The attachment to PMNs and the uptake of pneumococci by phagocytosis was stopped by centrifugation. The PMNs were fixed with $200 \mu \mathrm{PBS} / 1 \% \mathrm{FBS} / 1 \%$ paraformaldehyde. Binding and uptake of FITC-labelled bacteria were assessed by flow cytometry using a FACSCanto (Becton Dickinson). The mean fluorescence intensity (MFI) of the entire PMN population was recorded as a measure of bacterial binding and/or uptake. All experiments were performed independently three times.

J774 internalization assay. The number of viable intracellular pneumococci after phagocytosis by murine J744 macrophages was quantified by the antibiotic protection assay as described previously (Hermans et al., 2006). Briefly, the J774 cells were seeded in a 96-well format at a density of $1 \times 10^{5}$ per well. All bacterial suspensions were washed with PBS and diluted in a defined concentration in RPMI 1640, 1\% FCS medium. The confluent monolayers were then inoculated at an m.o.i. of 50. To synchronize the assay, the plates were centrifuged at $100 \mathrm{~g}$ for $4 \mathrm{~min}$. The number of c.f.u. inoculated per well was quantified by plating on BA plates. After $30 \mathrm{~min}$ of infection, the wells were washed four times with PBS to remove unbound pneumococci. To kill any extracellular bacteria, an incubation step with $1 \mathrm{ml}$ per well of DMEM ( $4.5 \mathrm{~g}$ glucose $1^{-1}$ ) and $1 \%$ FCS (no HEPES) containing gentamicin $\left(100 \mu \mathrm{g} \mathrm{ml}^{-1}\right)$ and penicillin $\mathrm{G}\left(100 \mathrm{IU} \mathrm{ml}^{-1}\right)$ was carried out for $1 \mathrm{~h}$. Intracellular pneumococci were recovered after three washes with DMEM by saponin-mediated lysis $(1 \% \mathrm{w} / \mathrm{v})$ for $10 \mathrm{~min}$ at $37{ }^{\circ} \mathrm{C}$ in $5 \% \mathrm{CO}_{2}$, and samples were plated for c.f.u. count. To differentiate between uptake and intracellular survival, time kinetic experiments were carried out as follows. Monolayers were infected for $30 \mathrm{~min}$ and then treated with gentamicin and penicillin $\mathrm{G}$ as described above. After removal of the antibiotics by washing, fresh medium was added, and the plate was incubated for 1,2 and $3 \mathrm{~h}$. Intracellular pneumococci were recovered by saponin-mediated lysis, and samples were plated for c.f.u. count. All experiments were performed at least in triplicate.

Statistical analysis. Comparisons of bacterial loads between wildtype and mutant bacterial strains were performed using Student's $t$ test (unpaired) for all in vitro assays and with the single infection of the colonization model. For the co-infection, a one sample $t$-test (with an arbitrary mean of 1) was used to calculate statistical significance for the CI scores. All statistical analyses were performed using GraphPad Prism version 4.0.

\section{RESULTS}

\section{Contribution of PpmA to pneumococcal colonization in vivo}

To assess the contribution of PpmA to pneumococcal virulence, we inactivated the ppmA gene in the D39, NCTC10319 and TIGR4 strains using insertion deletion mutagenesis. Immunoblot analysis of wild-type and $p p m A$ mutant cell lysates using anti-PpmA rabbit serum showed that PpmA was expressed in all wild-type strains, but not in generated mutants (data not shown). To examine the role of PpmA in colonization, both single and co-infections of wild-type and $\triangle p p m A$ mutant pneumococci were carried out using the murine colonization model. In this model, extended colonization of the nasopharynx is achieved by using a low volume $(10 \mu \mathrm{l})$ of inoculum. Some infection in the lungs was seen at the earlier time points, but, importantly, mice did not show any signs of invasive disease, and no mice died during the course of the experiment. After $192 \mathrm{~h}$, the D39 and TIGR4 wild-type strains colonized the nasopharynx at higher levels (4001000 times, respectively) than the NCTC10319 strain. A significant decrease in bacterial load of the nasopharynx was seen at $24 \mathrm{~h}$ post-infection and onwards with the D39 $\triangle p p m A$ mutant compared to its wild-type (Fig. 1a). By $192 \mathrm{~h}$, all six mice infected with D39 $\triangle p p m A$ had cleared the infection. Similar to D39 $\triangle p p m A$, TIGR4 $\triangle p p m A-$ infected mice had significant decreases in bacterial load after $48 \mathrm{~h}$ post-infection (Fig. 1b). Decrease in colonization with the NCTC10319 $\triangle p p m A$ mutant compared to its wildtype was seen as well, albeit only statistically significant in two out of six time points (Fig. 1c).

To validate the previous findings, we performed coinfections with each wild-type and its respective mutant over a period of 8 days. Again, overall colonization levels of the NCTC10319 wild-type were significantly lower than those of D39 and TIGR4 wild-type strains. In fact, the mice had cleared the infection caused by NCTC10319 wild-type and $p p m A$ mutant at $192 \mathrm{~h}$ post-infection in four out of six mice. Results of co-infection experiments confirmed the decreased ability of D39 $\Delta p p m A$ to colonize the nasopharynx, up to $60 \%$ less by the $192 \mathrm{~h}$ time point (Fig. 2a). Likewise, the TIGR4 $\triangle p p m A$ showed also a reduced colonization efficiency, at $192 \mathrm{~h}$ postinfection ( $35 \%$; Fig. 2b), while the NCTC10319 wildtype was unable to out-compete its isogenic ppmA mutant (Fig. 2c).

\section{Contribution of PpmA to pneumococcal adherence in vitro}

To assess the role of PpmA in pneumococcal adherence, we examined adherence of all three $S$. pneumoniae wild-type strains and their isogenic ppmA-deficient mutants to human pharyngeal epithelial Detroit 562 cells. Since previous studies have shown a negative effect of capsule expression on the adherence ability of $S$. pneumoniae (Bootsma et al., 2007; Hammerschmidt et al., 2005; Magee \& Yother, 2001; Selinger \& Reed, 1979; Talbot et al., 1996), we used non-encapsulated derivatives of all strains except NCTCC10319, which has a very low intrinsic level of capsule expression. In this way, all PpmA-expressing strains showed comparable levels of adherence (approx. $10^{5}$ c.f.u., data not shown). For the NCTC10319 and TIGR4 strains, a significant decrease in adherence of the 

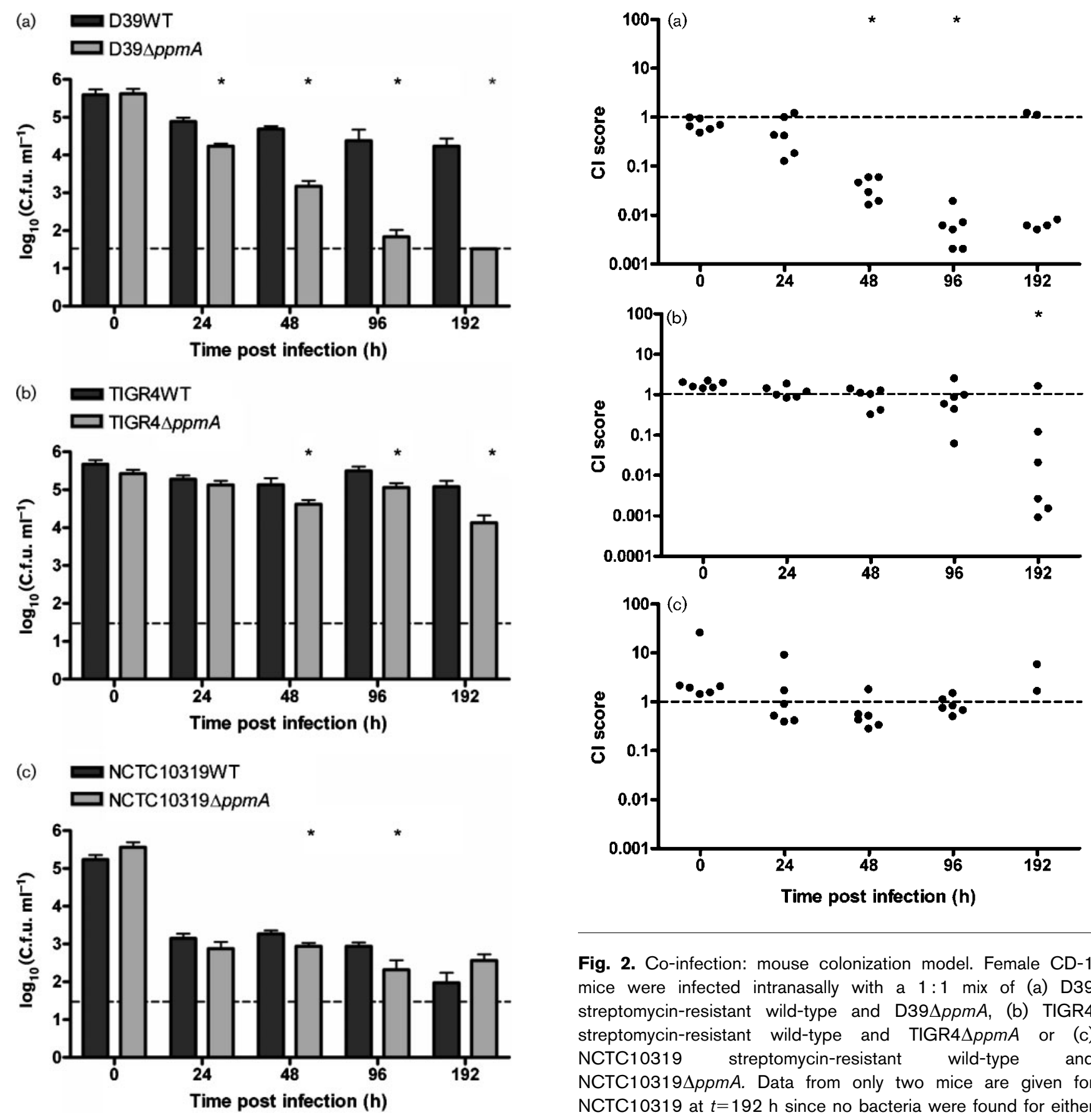

Fig. 2. Co-infection: mouse colonization model. Female CD-1 mice were infected intranasally with a 1:1 mix of (a) D39 streptomycin-resistant wild-type and D39 $\Delta p p m A$, (b) TIGR4 streptomycin-resistant wild-type and TIGR4 $\triangle p p m A$ or (c) NCTC10319 streptomycin-resistant wild-type and NCTC10319 $\Delta p p m A$. Data from only two mice are given for NCTC10319 at $t=192 \mathrm{~h}$ since no bacteria were found for either wild-type or ppmA mutant in the other four mice. Each dot represents one mouse. ${ }^{*}, P<0.05$.

Fig. 1. Single infection: mouse colonization model. Bacterial loads in the nasopharyngeal lavage fluid of mice intranasally infected with (a) D39 wild-type or D39 $\triangle p p m A$, (b) TIGR4 wildtype or TIGR4 $\triangle p p m A$, and (c) NCTC10319 wild-type or NCTC10319 $\Delta p p m A$. Five or six animals per group were used, with the following exceptions: NCTC10319 wild-type at $t=48 \mathrm{~h}$ $(n=4)$ and NCTC10319 $\Delta p p m A$ at $t=96 \mathrm{~h}(n=4)$. The dashed lines represent the lower limit of detection. ${ }^{*}, P<0.05$.

$\triangle p p m A$ mutants relative to wild-type was observed, namely $41 \%$ and $47 \%$ reduction, respectively (Fig. 3). Interestingly, no effect of the $p p m A$ deletion was observed

in the D39 background, suggesting that the contribution of PpmA to adherence is strain-specific.

\section{Effects of PpmA on pneumococcal host-protein binding}

Various host proteins derived from the extracellular matrix or serum are potential binding targets for S. pneumoniae, such as factor $\mathrm{H}$ and the secretory component (SC) of the 


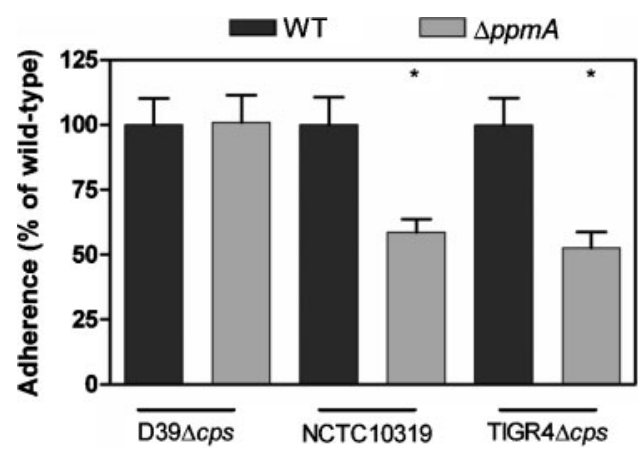

Fig. 3. In vitro adherence of pneumococcal strains to the human pharyngeal epithelial cell line Detroit 562. The adherence of the mutants is given as a percentage relative to the wild-type. *, $P<0.001$.

human polymeric immunoglobulin receptor (hpIgR). To assess whether deficiency in $p p m A$ affects the interaction of pneumococcal surface proteins with these cellular receptors or host proteins, we investigated the binding of $S$. pneumoniae NCTC10319 and its isogenic ppmA mutant to various immobilized extracellular matrix proteins. The pneumococcal mutant lacking $p p m A$ bound equally well to laminin, fibronectin, fibrinogen, lactoferrin, type IV collagen, plasminogen, serum IgA, human secretory component and reconstituted basement membrane (Fig. 4).

\section{Contribution of PpmA to pneumococcal uptake by host cells in vitro}

Phagocytosis by neutrophils and macrophages is an important process to eliminate bacteria. To investigate the role of PpmA in this process, we first explored the

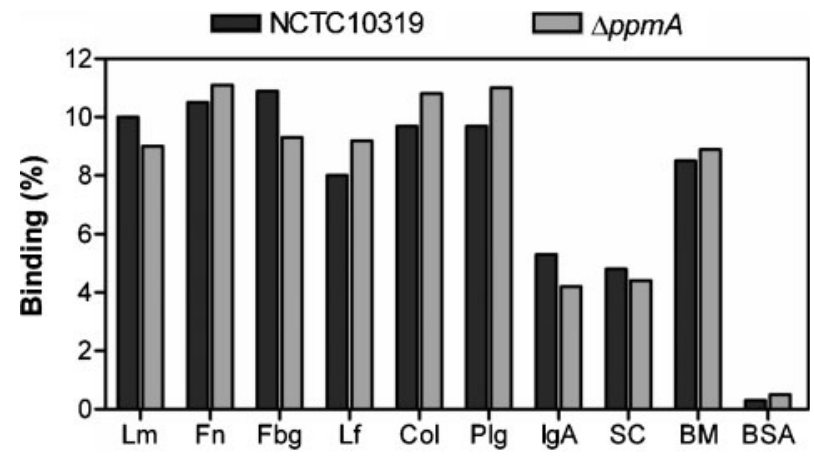

Fig. 4. Effect of $p p m A$ deletion on host-protein binding by pneumococci. Binding of FITC-labelled $S$. pneumoniae NCTC10319 and its isogenic ppmA mutant was assayed to immobilized host factors (Lm, laminin; Fn, fibronectin; Fbg, fibrinogen; Lf, lactoferrin; Col, type IV collagen; Plg, plasminogen; $\lg A$, serum IgA; SC, human secretory component; BM, Matrigel; and BSA, bovine serum albumin). interactions between the pneumococcus and human PMNs by flow cytometry using FITC-labelled D39 $\Delta c p s \Delta p p m A$ and NCTC10319 $\triangle p p m A$ and their respective wild-types. The ppmA mutants of both D39 $\Delta$ cps and NCTC10319 demonstrated a significantly increased association with human PMNs compared to their isogenic wild-types (Fig. 5a; $P$-values of 0.001 and 0.043 , respectively). Next, we examined the effect of PpmA on pneumococcal uptake by murine J774 professional phagocytes. A significant increase in the amount of internalized $\mathrm{D} 39 \Delta c p s \Delta p p m A$ compared to D39 $\Delta c p s$ was measured (Fig. 5b; $P$-value of 0.0004). However, no significant difference in uptake between the NCTC10319 wild-type or TIGR4 $\Delta c p s$ and their respective

(a)
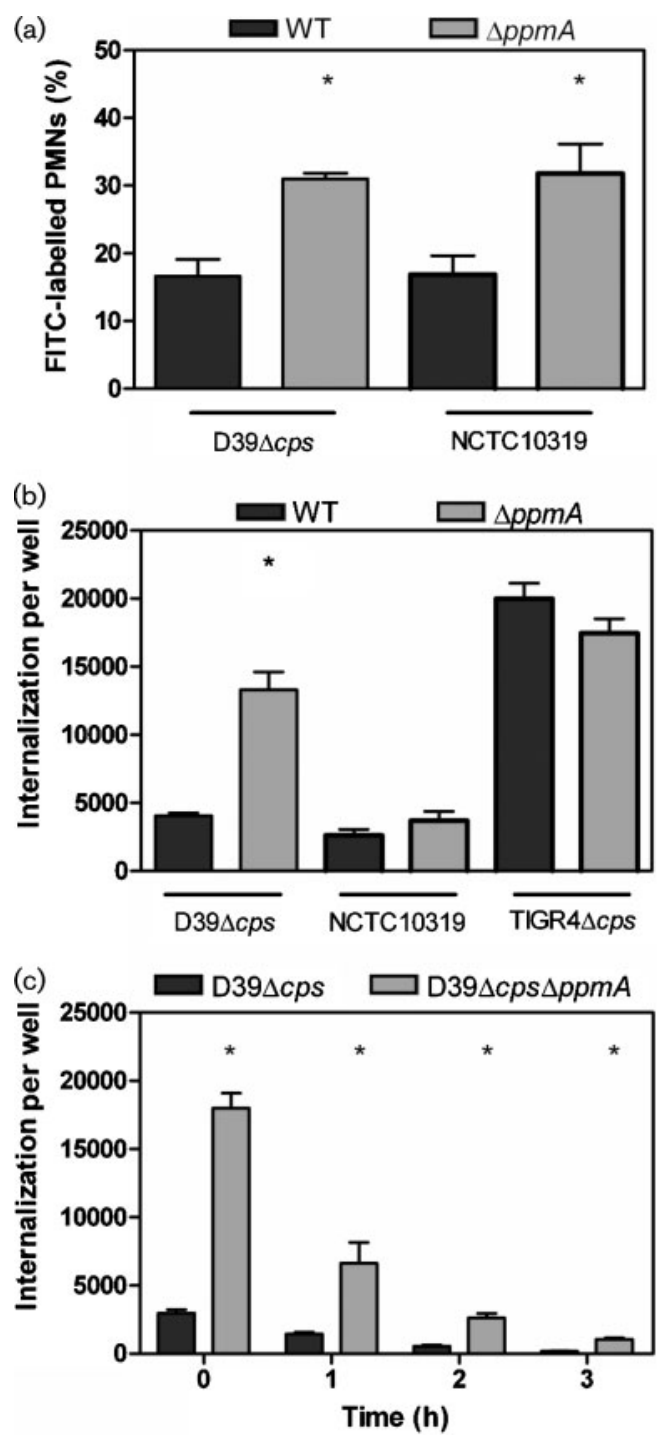

Fig. 5. Internalization and killing of $p p m A$-deficient pneumococci by PMNs and J774 macrophages. (a) Uptake of FITC-labelled pneumococci by PMNs. (b) Internalization of pneumococci by J774 macrophages. (c) Killing of pneumococci by J774 macrophages. *, $P<0.005$. 
$\triangle p p m A$ mutants by J774 cells was observed. To determine whether the observed difference is indeed due to increased uptake of the $p p m A$ mutant, and not the result of enhanced killing of intracellular wild-type, we examined intracellular survival using longer incubation periods of the bacteria and macrophages. This showed that the observed difference is indeed caused by enhanced uptake of D39 $\Delta p p m A$, as the rate of intracellular killing of wild-type and mutant was the same over time, with a mean of 5.3 -fold per hour (Fig. 5c).

\section{DISCUSSION}

Nasopharyngeal colonization is a prerequisite for the development of pneumococcal disease. Colonization and subsequent invasive infection of $S$. pneumoniae is dependent on the presence of various proteins targeting host components, such as pneumococcal surface adhesin A (PsaA) (Dintilhac et al., 1997; Romero-Steiner et al., 2003), pneumococcal adherence and virulence factor A (PavA) (Holmes et al., 2001; Lau et al., 2001) and PspC (Elm et al., 2004; Hammerschmidt et al., 2000; Rosenow et al., 1997; Zhang et al., 2000). Proper and efficient folding of such translocated or secreted proteins is crucial for their biochemical function and contribution to pneumococcal pathogenesis. PPIases are ubiquitous protein foldases, which have been shown to be required for virulence in various micro-organisms, such as L. pneumophila and $S$. pyogenes (Cianciotto et al., 1989; Cianciotto \& Fields, 1992; Lyon et al., 1998). S. pneumoniae produces two surfaceassociated PPIases, the lipoproteins SlrA and PpmA. Previously, we showed SlrA to be a functional cyclophilin contributing to the early stages of pneumococcal infection (Hermans et al., 2006). In this study, we examined the role of PpmA in pneumococcal virulence.

Our results clearly demonstrate that PpmA is involved in the early stages of the infection process in a strain-specific manner. The colonization ability of D $39 \Delta p p m A$ and TIGR4 $\triangle p p m A$ was found to be severely reduced compared to the wild-type strains in both single and co-infection models. However, even though deletion of $p p m A$ in the NCTC10319 strain resulted in lower levels of colonization compared to its wild-type, the difference was not statistically significant. The NCTC10319 strain was a poor colonizer of the mouse nasopharynx, which interferes with appropriate colonization read-out over time. Contributing to the poor colonization ability of NCTC10319 could be the fact that it produces very low amounts of capsule compared to the other two strains (Hammerschmidt et al., 2005). By testing defined mutants of the capsule locus, Magee \& Yother (2001) demonstrated that expression of a certain amount of capsule is required for efficient in vivo colonization. In line with this, pneumococci are known to undergo spontaneous phase variation, resulting in opaque and transparent colony variants that are characterized by high or low levels of capsule, respectively. Enhanced colonization and adherence are typically seen with the transparent phase variants, possibly by allowing better exposure to surface proteins that are important for adherence (Kim \& Weiser, 1998; Weiser et al., 1994). Furthermore, Briles et al. (2005) showed that mucosal tissues of mice support both the transparent and opaque populations of pneumococci. They hypothesized that local nasal mucosa may be required for maintenance of longterm carriage and by way of spontaneous phase shift, pneumococci create a reservoir in the mucosa giving sufficient opportunity to repopulate the nasal surface over time (Briles et al., 2005). Interestingly, PpmA was shown to be more prevalent in the transparent phenotype that is selected for during nasopharyngeal colonization (Overweg et al., 2000b).

Since adherence of $S$. pneumoniae is a prerequisite for successful colonization, it is likely that the observed role of PpmA in colonization is at least partially due to its contribution to adherence. The significant reduction in adherence observed with ppmA-deficient mutants of NCTC10319 and TIGR4 compared to their respective wild-type strains supports this hypothesis. However, no difference with the ppmA mutant was seen in the D39 background. This apparent discrepancy could, at least partly, be related to host-specific characteristics, i.e. differences between murine (in vivo colonization) and human (in vitro adherence) epithelial cells. S. pneumoniae binds via surfacelocated adhesins to various extracellular matrix and serum proteins, for example lactoferrin (Hammerschmidt et al., 1999), plasmin(ogen) (Bergmann et al., 2001, 2003, 2004) and immobilized fibronectin (Holmes et al., 2001; van der Flier et al., 1995). These adhesins and interactions have been shown to be involved in pneumococcal pathogenesis (Bergmann et al., 2003; Holmes et al., 2001; Shaper et al., 2004). However, our results indicate that PpmA does not contribute to the interactions of the pneumococcus with any of the aforementioned factors of the extracellular matrix and serum. Nonetheless, the effect of PpmA on adherence could still be mediated through host receptor/protein(s) not tested in this study.

Once colonization has been established, pneumococci can replicate and evade the defence mechanisms of the host's immune system. Macrophages and PMNs are essential players in eliminating bacterial infections. Significantly increased uptake by mouse J774 macrophages and human PMNs was seen with the D39 $\Delta p p m A$ mutant compared to the wild-type, whereas the deletion of $p p m A$ did not affect uptake in the TIGR4 background. No difference between intracellular killing of the D39 wild-type and ppmA mutant was observed. Our data suggest that in addition to colonization, PpmA contributes to immune evasion by preventing recognition and uptake by the macrophages at least in a D39 background.

PPIases are known to catalyse the cis-trans isomerization of peptidyl-prolyl bonds in vitro, but the physiological function of many of them is still unknown. The effects resulting from PPIase mutational inactivation can range 
from severe defects (Dartigalongue \& Raina, 1998; Lu et al., 1996) to none (Dolinski et al., 1997). Our data indicate that PpmA has a role in adherence, possibly by altering the surface composition of other bacterial surface proteins and adhesins that are involved in this process. In addition, the presence of PpmA appears to protect the pneumococcus against phagocytosis. So far, presumed pneumococcal protein target(s) of PpmA remain unknown and are the subject of current investigation. Because no PPIase activity has been detected for PpmA (Hermans et al., 2006), it is plausible that PpmA has evolved from a parvulin-like ancestor to perform functions other than peptidyl-prolyl isomerization.

The amino acid sequence of PpmA is conserved among strains, suggesting that not PpmA itself but the presumed target(s) of PpmA act in a strain-specific manner. Strain specificity of pneumococcal virulence factors has been described before. More than a decade ago it was demonstrated that the degree of protection of mice immunized with a pneumolysin toxoid derivative and subsequently challenged with various serotypes differs between strains (Alexander et al., 1994). Furthermore, in a study examining pneumococcal invasion of epithelial cells, where several hpIgR-expressing cell lines were used along with various non-encapsulated and encapsulated $S$. pneumoniae strains, the hpIgR-dependent invasion of epithelial cells by $S$. pneumoniae was both strain-specific and host cell type-specific (Brock et al., 2002). Finally, strain specificity is also present at the level of regulation of gene expression. For instance, the contribution to virulence of response regulator 09 (RR09) was found to vary between strains and routes of infection (Blue \& Mitchell, 2003). In line with this, Hendriksen et al. (2007) demonstrated strain-specific regulation of gene expression by RR09 when comparing D39 and TIGR4.

Alternatively, the targets of PpmA could differ between strains. The genomes of D39 and TIGR4 differ by approximately $10 \%$ of their genes (Hakenbeck et al., 2001). Several of these loci are surface-exposed and/or related to pathogenesis, and these differences may contribute to the diversity in virulence seen in animal infection models (Lanie et al., 2007; Tettelin et al., 2001). A prominent example is the rlrA pathogenicity islet, which is found in TIGR4 but not D39. The pilus encoded by this islet has been shown to contribute to colonization and invasive disease in vivo as well as adherence in vitro when using encapsulated strains (Barocchi et al., 2006). Whether any of these strain-specific loci actually are targets of PpmA and as such contribute to the differences observed in our study remains to be investigated.

In summary, we have demonstrated that PpmA has a role in pneumococcal pathogenesis in a murine colonization infection model. Altogether, the data suggest that the contribution of PpmA to colonization is not exclusively attributable to its role in adherence, but also to its role in preventing phagocytosis.

\section{ACKNOWLEDGEMENTS}

We thank Elles Simonetti, Christa van der Gaast, Marc Eleveld, and Dr Saskia van Selm for their technical assistance. H. J. B. and P. B. are supported by IOP Genomics grant IGE03002 from the Dutch Ministry of Economic Affairs.

\section{REFERENCES}

Alexander, J. E., Lock, R. A., Peeters, C. C., Poolman, J. T., Andrew, P. W., Mitchell, T. J., Hansman, D. \& Paton, J. C. (1994). Immunization of mice with pneumolysin toxoid confers a significant degree of protection against at least nine serotypes of Streptococcus pneumoniae. Infect Immun 62, 5683-5688.

Barocchi, M. A., Ries, J., Zogaj, X., Hemsley, C., Albiger, B., Kanth, A., Dahlberg, S., Fernebro, J., Moschioni, M. \& other authors (2006). A pneumococcal pilus influences virulence and host inflammatory responses. Proc Natl Acad Sci U S A 103, 2857-2862.

Bergmann, S., Rohde, M., Chhatwal, G. S. \& Hammerschmidt, S. (2001). alpha-Enolase of Streptococcus pneumoniae is a plasmin(ogen)-binding protein displayed on the bacterial cell surface. $\mathrm{Mol}$ Microbiol 40, 1273-1287.

Bergmann, S., Wild, D., Diekmann, O., Frank, R., Bracht, D., Chhatwal, G. S. \& Hammerschmidt, S. (2003). Identification of a novel plasmin(ogen)-binding motif in surface displayed alpha-enolase of Streptococcus pneumoniae. Mol Microbiol 49, 411-423.

Bergmann, S., Rohde, M. \& Hammerschmidt, S. (2004). Glyceraldehyde-3-phosphate dehydrogenase of Streptococcus pneumoniae is a surface-displayed plasminogen-binding protein. Infect Immun 72, 2416-2419.

Blue, C. E. \& Mitchell, T. J. (2003). Contribution of a response regulator to the virulence of Streptococcus pneumoniae is strain dependent. Infect Immun 71, 4405-4413.

Bogaert, D., de Groot, R. \& Hermans, P. W. (2004). Streptococcus pneumoniae colonisation: the key to pneumococcal disease. Lancet Infect Dis 4, 144-154.

Bootsma, H. J., Egmont-Petersen, M. \& Hermans, P. W. (2007). Analysis of the in vitro transcriptional response of human pharyngeal epithelial cells to adherent Streptococcus pneumoniae: evidence for a distinct response to encapsulated strains. Infect Immun 75, 54895499.

Briles, D. E., Novak, L., Hotomi, M., van Ginkel, F. W. \& King, J. (2005). Nasal colonization with Streptococcus pneumoniae includes subpopulations of surface and invasive pneumococci. Infect Immun 73, 6945-6951.

Brock, S. C., McGraw, P. A., Wright, P. F. \& Crowe, J. E., Jr (2002). The human polymeric immunoglobulin receptor facilitates invasion of epithelial cells by Streptococcus pneumoniae in a strain-specific and cell type-specific manner. Infect Immun 70, 5091-5095.

Burghout, P., Bootsma, H. J., Kloosterman, T. G., Bijlsma, J. J., de Jongh, C. E., Kuipers, O. P. \& Hermans, P. W. (2007). Search for genes essential for pneumococcal transformation: the RadA DNA repair protein plays a role in genomic recombination of donor DNA. J Bacteriol 189, 6540-6550.

Cianciotto, N. P. \& Fields, B. S. (1992). Legionella pneumophila mip gene potentiates intracellular infection of protozoa and human macrophages. Proc Natl Acad Sci U S A 89, 5188-5191.

Cianciotto, N. P., Eisenstein, B. I., Mody, C. H., Toews, G. B. \& Engleberg, N. C. (1989). A Legionella pneumophila gene encoding a species-specific surface protein potentiates initiation of intracellular infection. Infect Immun 57, 1255-1262. 
Cundell, D. R., Gerard, N. P., Gerard, C., Idanpaan-Heikkila, I. \& Tuomanen, E. I. (1995). Streptococcus pneumoniae anchor to activated human cells by the receptor for platelet-activating factor. Nature 377, 435-438.

Dartigalongue, C. \& Raina, S. (1998). A new heat-shock gene, ppiD, encodes a peptidyl-prolyl isomerase required for folding of outer membrane proteins in Escherichia coli. EMBO J 17, 3968-3980.

Dintilhac, A., Alloing, G., Granadel, C. \& Claverys, J. P. (1997). Competence and virulence of Streptococcus pneumoniae: Adc and PsaA mutants exhibit a requirement for $\mathrm{Zn}$ and $\mathrm{Mn}$ resulting from inactivation of putative ABC metal permeases. Mol Microbiol 25, 727739.

Dolinski, K., Muir, S., Cardenas, M. \& Heitman, J. (1997). All cyclophilins and FK506 binding proteins are, individually and collectively, dispensable for viability in Saccharomyces cerevisiae. Proc Natl Acad Sci U S A 94, 13093-13098.

Drouault, S., Anba, J., Bonneau, S., Bolotin, A., Ehrlich, S. D. \& Renault, P. (2002). The peptidyl-prolyl isomerase motif is lacking in PmpA, the PrsA-like protein involved in the secretion machinery of Lactococcus lactis. Appl Environ Microbiol 68, 3932-3942.

Elm, C., Braathen, R., Bergmann, S., Frank, R., Vaerman, J. P., Kaetzel, C. S., Chhatwal, G. S., Johansen, F. E. \& Hammerschmidt, S. (2004). Ectodomains 3 and 4 of human polymeric Immunoglobulin receptor (hpIgR) mediate invasion of Streptococcus pneumoniae into the epithelium. J Biol Chem 279, 6296-6304.

Hakenbeck, R., Balmelle, N., Weber, B., Gardès, C., Keck, W. \& de Saizieu, A. (2001). Mosaic genes and mosaic chromosomes: intraand interspecies genomic variation of Streptococcus pneumoniae. Infect Immun 69, 2477-2486.

Hammerschmidt, S. (2006). Adherence molecules of pathogenic pneumococci. Curr Opin Microbiol 9, 12-20.

Hammerschmidt, S., Bethe, G., Remane, P. H. \& Chhatwal, G. S. (1999). Identification of pneumococcal surface protein $A$ as a lactoferrin-binding protein of Streptococcus pneumoniae. Infect Immun 67, 1683-1687.

Hammerschmidt, S., Tillig, M. P., Wolff, S., Vaerman, J. P. \& Chhatwal, G. S. (2000). Species-specific binding of human secretory component to SpsA protein of Streptococcus pneumoniae via a hexapeptide motif. Mol Microbiol 36, 726-736.

Hammerschmidt, S., Wolff, S., Hocke, A., Rosseau, S., Muller, E. \& Rohde, M. (2005). Illustration of pneumococcal polysaccharide capsule during adherence and invasion of epithelial cells. Infect Immun 73, 4653-4667.

Hava, D. L. \& Camilli, A. (2002). Large-scale identification of serotype 4 Streptococcus pneumoniae virulence factors. Mol Microbiol 45, 13891406.

Hendriksen, W. T., Silva, N., Bootsma, H. J., Blue, C. E., Paterson, G. K., Kerr, A. R., de Jong, A., Kuipers, O. P., Hermans, P. W. \& Mitchell, T. J. (2007). Regulation of gene expression in Streptococcus pneumoniae by response regulator 09 is strain dependent. J Bacteriol 189, 1382-1389.

Hendriksen, W. T., Bootsma, H. J., Estevao, S., Hoogenboezem, T., de Jong, A., de Groot, R., Kuipers, O. P. \& Hermans, P. W. (2008), CodY of Streptococcus pneumoniae: link between nutritional gene regulation and colonization. J Bacteriol 190, 590-601.

Hermans, P. W., Adrian, P. V., Albert, C., Estevao, S., Hoogenboezem, T., Luijendijk, I. H., Kamphausen, T. \& Hammerschmidt, S. (2006). The streptococcal lipoprotein rotamase A (SlrA) is a functional peptidyl-prolyl isomerase involved in pneumococcal colonization. J Biol Chem 281, 968-976.

Holmes, A. R., McNab, R., Millsap, K. W., Rohde, M., Hammerschmidt, S., Mawdsley, J. L. \& Jenkinson, H. F. (2001). The pavA gene of Streptococcus pneumoniae encodes a fibronectin- binding protein that is essential for virulence. Mol Microbiol 41, 13951408.

Jansen, W. T., Gootjes, J., Zelle, M., Madore, D. V., Verhoef, J., Snippe, H. \& Verheul, A. F. (1998). Use of highly encapsulated Streptococcus pneumoniae strains in a flow-cytometric assay for assessment of the phagocytic capacity of serotype-specific antibodies. Clin Diagn Lab Immunol 5, 703-710.

Kerr, A. R., Adrian, P. V., Estevão, S., de Groot, R., Alloing, G., Claverys, J. P., Mitchell, T. J. \& Hermans, P. W. (2004). The Ami-AliA/ AliB permease of Streptococcus pneumoniae is involved in nasopharyngeal colonization but not in invasive disease. Infect Immun 72, 3902-3906.

Kim, J. O. \& Weiser, J. N. (1998). Association of intrastrain phase variation in quantity of capsular polysaccharide and teichoic acid with the virulence of Streptococcus pneumoniae. J Infect Dis 177, 368-377.

Lanie, J. A., Ng, W. L., Kazmierczak, K. M., Andrzejewski, T. M., Davidsen, T. M., Wayne, K. J., Tettelin, H., Glass, J. I. \& Winkler, M. E. (2007). Genome sequence of Avery's virulent serotype 2 strain D39 of Streptococcus pneumoniae and comparison with that of unencapsulated laboratory strain R6. J Bacteriol 189, 38-51.

Lau, G. W., Haataja, S., Lonetto, M., Kensit, S. E., Marra, A., Bryant, A. P., McDevitt, D., Morrison, D. A. \& Holden, D. W. (2001). A functional genomic analysis of type 3 Streptococcus pneumoniae virulence. Mol Microbiol 40, 555-571.

Lazar, S. W. \& Kolter, R. (1996). SurA assists the folding of Escherichia coli outer membrane proteins. J Bacteriol 178, 1770-1773.

Lu, K. P., Hanes, S. D. \& Hunter, T. (1996). A human peptidyl-prolyl isomerase essential for regulation of mitosis. Nature 380, 544-547.

Lyon, W. R., Gibson, C. M. \& Caparon, M. G. (1998). A role for trigger factor and an Rgg-like regulator in the transcription, secretion and processing of the cysteine proteinase of Streptococcus pyogenes. EMBO $J$ 17, 6263-6275.

Magee, A. D. \& Yother, J. (2001). Requirement for capsule in colonization by Streptococcus pneumoniae. Infect Immun 69, 37553761.

Nelson, A. L., Roche, A. M., Gould, J. M., Chim, K., Ratner, A. J. \& Weiser, J. N. (2007). Capsule enhances pneumococcal colonization by limiting mucus-mediated clearance. Infect Immun 75, 83-90.

Overweg, K., Kerr, A., Sluijter, M., Jackson, M. H., Mitchell, T. J., de Jong, A. P., de Groot, R. \& Hermans, P. W. (2000a). The putative proteinase maturation protein A of Streptococcus pneumoniae is a conserved surface protein with potential to elicit protective immune responses. Infect Immun 68, 4180-4188.

Overweg, K., Pericone, C. D., Verhoef, G. G., Weiser, J. N., Meiring, H. D., de Jong, A. P., de Groot, R. \& Hermans, P. W. (2000b). Differential protein expression in phenotypic variants of Streptococcus pneumoniae. Infect Immun 68, 4604-4610.

Rahfeld, J. U., Rucknagel, K. P., Schelbert, B., Ludwig, B., Hacker, J., Mann, K. \& Fischer, G. (1994). Confirmation of the existence of a third family among peptidyl-prolyl cis/trans isomerases. Amino acid sequence and recombinant production of parvulin. FEBS Lett 352, 180-184.

Romero-Steiner, S., Pilishvili, T., Sampson, J. S., Johnson, S. E., Stinson, A., Carlone, G. M. \& Ades, E. W. (2003). Inhibition of pneumococcal adherence to human nasopharyngeal epithelial cells by anti-PsaA antibodies. Clin Diagn Lab Immunol 10, 246-251.

Rosenow, C., Ryan, P., Weiser, J. N., Johnson, S., Fontan, P., Ortqvist, A. \& Masure, H. R. (1997). Contribution of novel choline-binding proteins to adherence, colonization and immunogenicity of Streptococcus pneumoniae. Mol Microbiol 25, 819-829.

Rouviere, P. E. \& Gross, C. A. (1996). SurA, a periplasmic protein with peptidyl-prolyl isomerase activity, participates in the assembly of outer membrane porins. Genes Dev 10, 3170-3182. 
Selinger, D. S. \& Reed, W. P. (1979). Pneumococcal adherence to human epithelial cells. Infect Immun 23, 545-548.

Shaper, M., Hollingshead, S. K., Benjamin, W. H., Jr \& Briles, D. E. (2004). PspA protects Streptococcus pneumoniae from killing by apolactoferrin, and antibody to PspA enhances killing of pneumococci by apolactoferrin. Infect Immun 72, 5031-5040.

Swords, W. E., Buscher, B. A., Ver Steeg, I. K., Preston, A., Nichols, W. A., Weiser, J. N., Gibson, B. W. \& Apicella, M. A. (2000). Nontypeable Haemophilus influenzae adhere to and invade human bronchial epithelial cells via an interaction of lipooligosaccharide with the PAF receptor. Mol Microbiol 37, 13-27.

Talbot, U. M., Paton, A. W. \& Paton, J. C. (1996). Uptake of Streptococcus pneumoniae by respiratory epithelial cells. Infect Immun 64, 3772-3777.

Tettelin, H., Nelson, K. E., Paulsen, I. T., Eisen, J. A., Read, T. D., Peterson, S., Heidelberg, J., DeBoy, R. T., Haft, D. H. \& other authors (2001). Complete genome sequence of a virulent isolate of Streptococcus pneumoniae. Science 293, 498-506. van der Flier, M., Chhun, N., Wizemann, T. M., Min, J., McCarthy, J. B. \& Tuomanen, E. I. (1995). Adherence of Streptococcus pneumoniae to immobilized fibronectin. Infect Immun 63, 4317-4322.

Vitikainen, M., Lappalainen, I., Seppala, R., Antelmann, H., Boer, H., Taira, S., Savilahti, H., Hecker, M., Vihinen, M. \& other authors (2004). Structure-function analysis of PrsA reveals roles for the parvulin-like and flanking $\mathrm{N}$ - and C-terminal domains in protein folding and secretion in Bacillus subtilis. J Biol Chem 279, 1930219314.

Weiser, J. N., Austrian, R., Sreenivasan, P. K. \& Masure, H. R. (1994). Phase variation in pneumococcal opacity: relationship between colonial morphology and nasopharyngeal colonization. Infect Immun 62, 2582-2589.

Zhang, J. R., Mostov, K. E., Lamm, M. E., Nanno, M., Shimida, S., Ohwaki, M. \& Tuomanen, E. (2000). The polymeric immunoglobulin receptor translocates pneumococci across human nasopharyngeal epithelial cells. Cell 102, 827-837.

Edited by: T. J. Mitchell 